\title{
Harlequin syndrome
}

INSERM

\section{Source}

INSERM. (1999). Orphanet: an online rare disease and orphan drug data base. Harlequin syndrome. ORPHA:199282

Harlequin syndrome (HSD) is an autonomic disorder occurring at any age and characterized by unilateral flushing and sweating, involving the face and sometimes arm and chest, in condition of thermal, exercise or emotional stress without sympathetic ocular manifestations. However, tonic pupils, parasympathetic oculomotor lesion and pre- or postgang lionic sudomotor sympathetic deficit can rarely occur. 